\title{
Le pouvoir calorifique supérieur d'espèces forestières méditerranéennes
}

\author{
Jacqueline DOAT et J.Ch. VALETTE * \\ avec la collaboration technique de D. Askri, L. Caumartin, M. Bettachini *, M. Moro* \\ C.T.F.T., Division Cellulose-Chimie, \\ 45 bis, avenue de la Belle-Gabrielle, F 94130 Nogent-sur-Marne \\ * I.N.R.A., Station de Sylviculture méditerranéenne, \\ avenue A.-Vivaldi, F 84000 Avignon
}

\section{Résumé}

Le pouvoir calorifique supérieur (P.C.S.) est le maximum d'énergie que peut dégager une combustion. Il conduit au bilan énergétique d'un incendie de forêt.

Rapporté au poids de matière sèche ou au poids de la seule matière organique, le P.C.S. permet de hiérarchiser les végétaux selon leur caractère énergétique. Bien que les P.C.S. croissent avec la lignification des tissus, ils conduisent au classement par ordre décroissant :

— feuilles de bruyère arborescente,

— feuilles de pin d'Alep,

- feuilles d'arbousier et pousses terminales d'ajonc épineux,

- feuilles de chêne vert et de chêne kermès.

Les P.C.S. de ces espèces méditerranéennes sont supérieurs ou al moins égaux à ceux d'espèces de régions plus tempérées.

\section{1. - Introduction}

Chaque année en région méditerranéenne française - dans les six départements de la région Provence-Alpes-Côte d'Azur, les cinq départements de la région Languedoc-Roussillon, les deux départements de la région Corse et l'Ardèche — près de 40000 ha de formations forestières, plus ou moins dégradées, sont la proie des flammes. Une meilleure connaissance des facteurs ayant une influence sur l'éclosion et sur la propagation de l'incendie devrait conduire à une meilleure prévention et à une économie sur les moyens de lutte.

En particulier, la connaissance de l'énergie dégagée lors d'un incendie et celle présente, à l'état potentiel, dans la formation forestière est indispensable. La mesure de la phytomasse aérienne du combustible et celle de la quantité de chaleur dégagée par unité de masse lors de la combustion constituent une approche satisfaisante de cette connaissance. 
Si des mesures classiques permettent de répondre à la première préoccupation, seul le «débit calorifique», contribution d'un échantillon à un dégagement énergétique global, donne la quantité de chaleur dégagée en fonction du degré de destruction du végétal. La méthode décrite par E.E. Sмiтн (1972) pour l'étude des matériaux de construction est pour l'instant la seule utilisable malgré ses imperfections. Une méthode adaptée aux végétaux est en cours d'élaboration à la station de sylviculture méditerranéenne.

La mesure du pouvoir calorifique supérieur - P.C.S. - reste donc actuellement la seule voie pour quantifier cette énergie potentielle car, ainsi que l'écrit W.A. Hough (1969) «bien qu'une combustion complète n'ait jamais lieu au cours d'un incendie de forêt, la mesure du P.C.S. fournit une donnée de base pour déterminer le débit calorifique potentiel dans de tels incendies ».

Le pouvoir calorifique d'un corps est la quantité totale de chaleur dégagée par la combustion complète de l'unité de poids de ce corps. Il est dit «supérieur» lorsque la combustion est effectuée à volume constant et que l'eau formée au cours de la combustion est condensée. Il est dit «inférieur» lorsque la combustion est effectuée à pression constante - la plupart du temps à la pression atmosphérique et qu'ainsi l'eau se vaporise librement. Les combustibles sont habituellement caractérisés par leurs P.C.S. :

- la mesure est normalisée par l'AFNOR ;

- les valeurs sont les maxima des énergies disponibles et satisfont aux critères de reproductibilité ;

- les mesures directes du P.C.I. sont plus aléatoires.

Comme l'indique G. Heım (1974), les P.C.S. d'espèces végétales ont été mesurés, aussi bien en France qu'à l'étranger, surtout par des écologistes "pour le calcul du rendement énergétique et la production primaire ainsi que pour l'étude des chaînes trophiques ».

En 1969, F.B. Golley conclut que les espèces de la mangrove ont généralement des P.C.S. plus élevés que les autres espèces tropicales, que ces dernières ont des P.C.S. plus faibles que ceux des espèces des régions tempérées ou alpines, alors qu'en 1977 J. DoAT prouve que les P.C.S. des bois des espèces tropicales sont, en général, plus élevés que ceux des bois des feuillus européens.

Toujours en 1969, W.A. Hough publie une étude portant sur toute une série d'espèces forestières du Sud et de l'Est des U.S.A. Il propose une classification des combustibles forestiers en quatre catégories:

P.C.S. inférieur à $4000 \mathrm{cal} / \mathrm{g}(16,7 \mathrm{~kJ} / \mathrm{g})$.

P.C.S. compris entre 4000 et $4500 \mathrm{cal} / \mathrm{g}(16,7$ et $18,8 \mathrm{~kJ} / \mathrm{g})$.

P.C.S. compris entre 4500 et $5000 \mathrm{cal} / \mathrm{g}(18,8$ et $20,9 \mathrm{~kJ} / \mathrm{g})$.

P.C.S. supérieur à $5000 \mathrm{cal} / \mathrm{g}(20,9 \mathrm{~kJ} / \mathrm{g})$.

D.A. BoAG \& J.W. KiCEnIUK (1968) montrent qu'à une faible teneur protéinique des aiguilles du pin de Murray correspond un P.C.S. élevé. W. Lafctiek (1973) et son équipe mettent en relation une importante accumulation de lipides dans les feuilles âgées et les tiges de Loiseleuria procuminens (L.) Desv. avec un P.C.S. élevé. 
Enfin, G. Heim (1974) fournit les P.C.S. de 37 espèces autochtones de la région de Montpellier, prélevées surtout au printemps; il met en parallèle « les fluctuations des valeurs énergétiques et la composition chimique des tissus et des organes».

La présente étude est le fruit d'une active coopération: P.C.S. ;

- de la division cellulose-chimie du C.T.F.T. qui a effectué les mesures de

- de la station de sylviculture méditerranéenne de l'I.N.R.A. qui a effectué les prélèvements d'échantillons et l'analyse des résultats.

Elle présente les P.C.S. des principales espèces forestières méditerranéennes, met en évidence les différences spécifiques, fait apparaître les variations saisonnières, permet enfin le calcul de la «puissance du front de feu» par application de la formule de BYram.

\section{2. - Méthodes et matériels}

\section{1. - Définitions}

Le P.C.S. est mesuré dans un calorimètre adiabatique et s'exprime classiquement en calories $\left(^{*}\right)$ par gramme de matière sèche. Dans le système d'unités international, la chaleur étant exprimée en Joules, le P.C.S. s'exprime en $\mathrm{J} / \mathrm{g}$ ou en $\mathrm{kJ} / \mathrm{g}$.

Le pouvoir calorifique inférieur P.C.I. se déduit du P.C.S. par application d'une formule conventionnelle mentionnée dans la norme AF M03-005 qui revient, pour les combustibles végétaux dont la teneur en hydrogène est de lordre de 6 p. 100, à déduire $320 \mathrm{cal} / \mathrm{g}(1340 \mathrm{~J} / \mathrm{g})$ du P.C.S.

Cette valeur est très proche de celle retenue par W.A. Hough (1969) qui cite les travaux de BYRAM.

Le P.C.S. comme le P.C.I. peuvent être rapportés à la matière verte par la formule que propose Nierat, cité par J. Doat (1977).

$$
\operatorname{Pe}=P_{0} \times \frac{100-E}{100}-6 E
$$

E : teneur en eau, en pourcentage de la matière verte.

Pe : pouvoir calorifique de la matière à une teneur en eau $\mathrm{E}$.

Po : pouvoir calorifique de la matière sèche.

(*) Une calorie est la quantité de chaleur nécessaire pour élever de 1 'C la température d'un corps dont la chaleur massique est égale à celle de l'eau à $15^{\circ} \mathrm{C}$ sous la pression atmosphérique normale. 1 calorie $=4,1855$ joules. 
Enfin, les pouvoirs calorifiques peuvent être rapportés au poids de la seule matière organique par application de la formule suivante :

$$
\text { Porg. }=\frac{100 \text { Ptot. }}{100-\mathrm{Ce}}
$$

Porg. : pouvoir calorifique de la matière organique.

Ptot. : pouvoir calorifique de la matière organique et des éléments minéraux.

$\mathrm{Ce} \quad$ : teneur en cendres, en pourcentage de la matière sèche.

\section{2. - Méthodologie}

Après passage à l'étuve durant $18 \mathrm{~h}$ à $105^{\circ} \mathrm{C}$, la mesure est faite sur environ $1,8 \mathrm{~g}$ de matière sèche broyée puis comprimée en une pastille à l'aide d'une presse à main. Lorsqu'il s’agit de tiges dures lignifiées ou de bois, on utilise également le matériau sec fractionné en portions de la taille d'une demi-allumette.

La pression d'oxygène de la bombe calorimétrique est portée à 30 bars après introduction de l'échantillon : combustion en atmosphère sur-oxygénée, donc complète. La bombe est alors placée dans le calorimètre adiabatique de Gallempkamp. En fin de combustion, déclenchée électriquement, une fois l'équilibre thermique établi la température de leau du calorimètre est mesurée au 5 millièmes de degré Celsius. Une mesure préliminaire, à l'aide d'acide benzoïque rigoureusement pur de P.C.S. égal à $6319 \mathrm{cal} / \mathrm{g}(26448 \mathrm{~J} / \mathrm{g})$ permet de calculer la «valeur en eau » du calorimètre.

La précision des mesures du P.C.S. est de l'ordre de 1 p. 100 au seuil de probabilité de 95 p. 100 . Pour une même espèce, deux mesures sont systématiquement effectuées, une troisième étant réalisée en cas d'écart supérieur à la précision entre les deux premières.

Malgré la rapidité de la manipulation et les précautions prises, les échantillons se rechargent légèrement en eau entre la sortie de l'étuve et la mise à feu de la bombe. Le biais serait de l'ordre de la précision de la mesure.

\section{3. - Les végétaux}

L'étude de l'année 1979 a porté sur des espèces arborées et arbustives de Provence calcaire et de Provence cristalline.

Le tableau $n^{\prime \prime} 1$ comporte aussi l'indication de la partie du végétal systématiquement étudiée.

La végétation est collectée à la fin de chaque mois et le stade phénologique des végétaux est systématiquement noté. Les rameaux feuillés sont prélevés, autant que faire se peut, sur les mêmes individus pour limiter au maximum les sources d'hétérogénéité de l'échantillonnage. 


\section{Tableau 1}

Matériel végétal utilisé

Studied vegetation

\begin{tabular}{|c|c|c|c|c|c|}
\hline \multirow{2}{*}{ Espèces étudiées } & \multicolumn{2}{|c|}{ Provence } & \multicolumn{2}{|c|}{ Formation } & \multirow{2}{*}{$\begin{array}{l}\text { Partie } \\
\text { du végétal }\end{array}$} \\
\hline & Calcaire & Cristall. & Arborée & Arbustive & \\
\hline $\begin{array}{l}\text { Erica arborea } \mathrm{L} . \\
\text { Bruyère arborescente }\end{array}$ & & $x$ & & $x$ & Feuilles \\
\hline $\begin{array}{l}\text { Pinus halepensis Mill. } \\
\text { Pin d'Alep } \ldots \ldots \ldots \ldots \ldots \ldots\end{array}$ & $x$ & & $x$ & & Aiguilles \\
\hline $\begin{array}{l}\text { Ulex parviflorus Pourret } \\
\text { Ajonc épineux } \ldots \ldots \ldots \ldots\end{array}$ & $x$ & & & $x$ & Sommités * \\
\hline $\begin{array}{l}\text { Arbutus unedo } \mathbf{L} \text {. } \\
\text { Arbousier } \quad \ldots . .\end{array}$ & & $\times$ & & $x$ & Feuilles \\
\hline $\begin{array}{l}\text { Quercus ilex } \mathrm{L} \text {. } \\
\text { Chêne vert } \ldots\end{array}$ & $x$ & & $x$ & & Feuilles \\
\hline $\begin{array}{l}\text { Quercus coccifera } \mathrm{L} . \\
\text { Chêne kermès } \ldots \ldots \ldots \ldots \ldots\end{array}$ & $x$ & & & $x$ & Feuilles \\
\hline
\end{tabular}

* Sommités : extrêmités des rameaux latéraux et terminaux.

Des lots sont ensuite constitués en veillant :

- à ne conserver que les feuilles de chênes, de la bruyère et de l'arbousier ;

- à enlever la gaine des aiguilles de pin ;

- à broyer le matériel végétal après un premier passage à l'étuve, opération indispensable pour homogénéiser les échantillons d'ajonc épineux.

\section{3. - Résultats et discussion}

L'examen du tableau $n^{\circ} 2$ montre que, bien que le P.C.S. évolue au cours de l'année, il atteint des valeurs caractéristiques pour chacune des six espèces.

\section{1. - Evolution du pouvoir calorifique supérieur}

Le tableau n" 2 fournit pour chacun des douze mois de l'année 1979, les deux mesures retenues pour chaque espèce du tableau $n^{\circ} 1$. La figure 1 matérialise cette évolution. 
Tableau 2

Evolution du P.C.S. au cours de l'année (cal/g)

Annual variation of the high calorific value ( $\mathrm{cal} / \mathrm{g})$

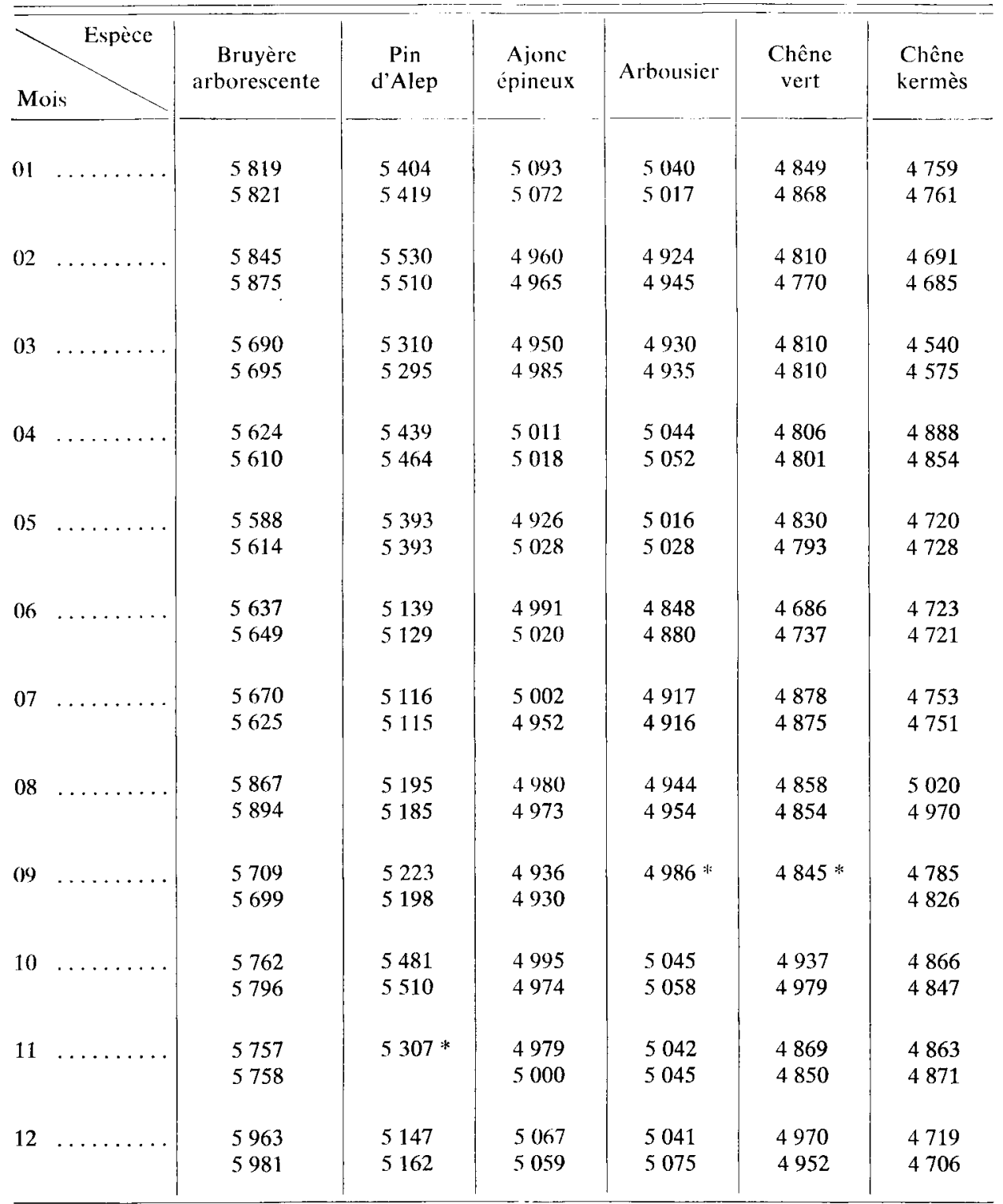

* Valeurs absentes ou aberrantes, remplacées par la moyenne des 11 couples de P.C.S. 


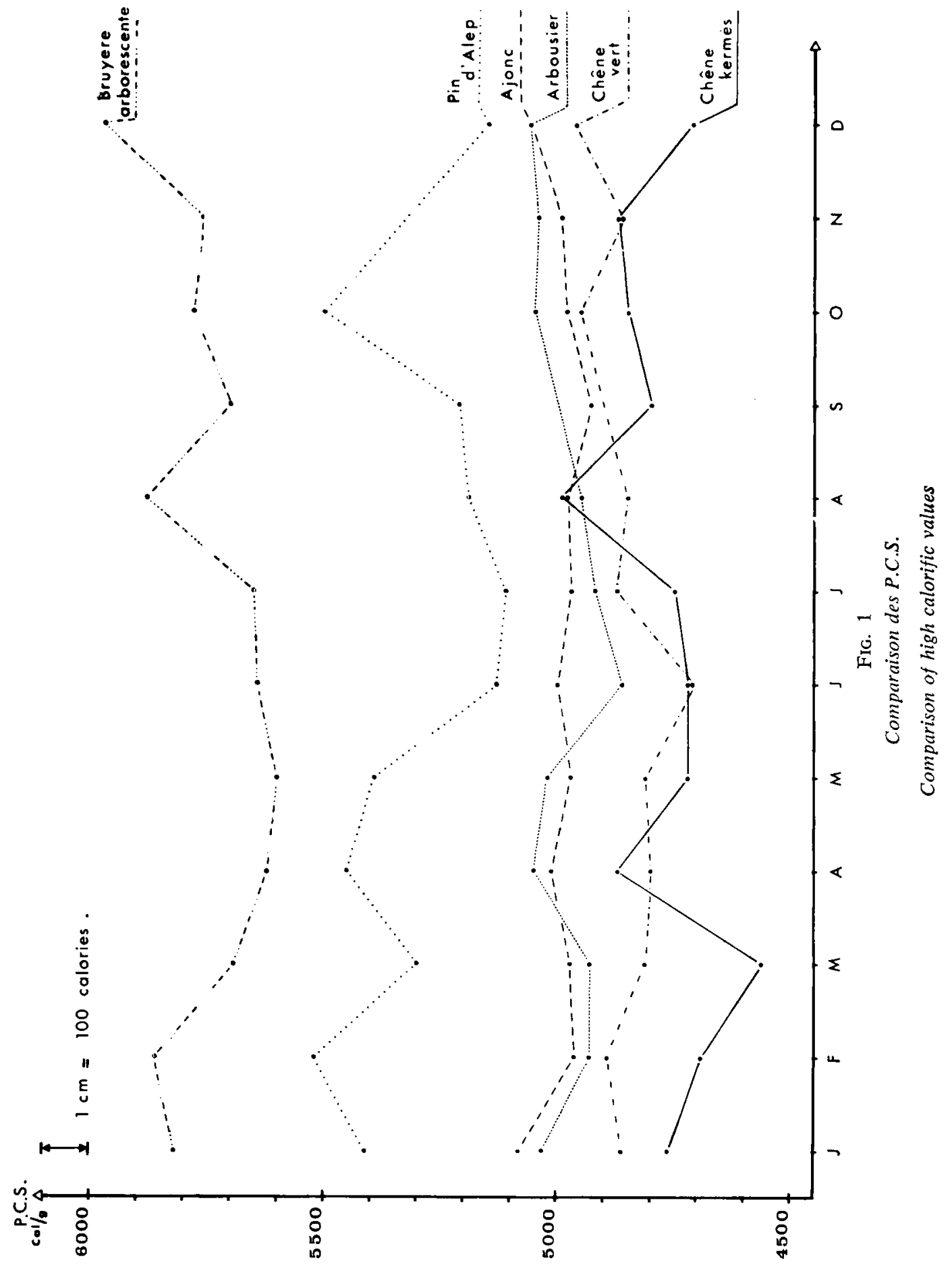




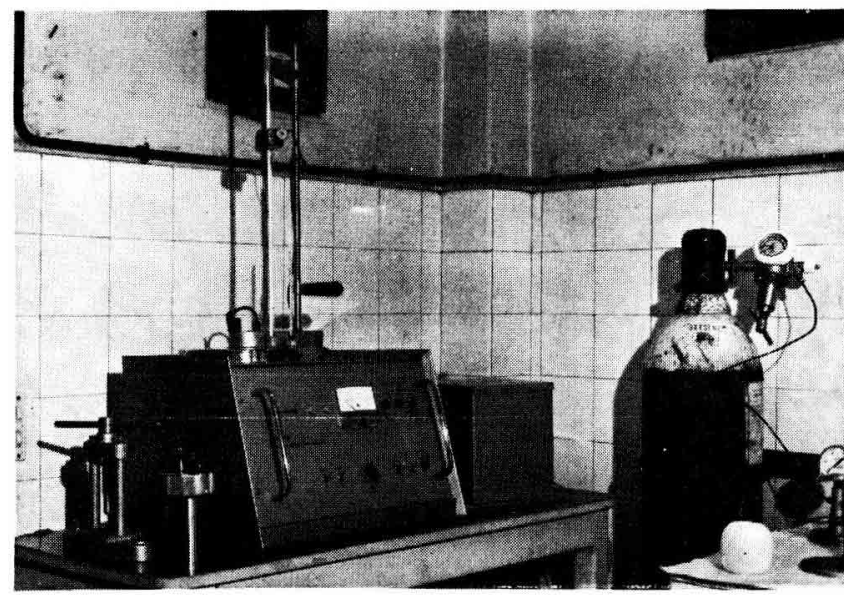

Рното 1

Le calorimètre adiabatique (et la bombe)

utilisés pour les mesures des pouvoirs calorifiques supérieurs

Adiabatic calorimeter for calorific value measurements

Au cours des quatre premiers mois, les échantillons sont prélevés sur des pousses d'un an, apparues en 1978. A partir du mois de mai 1979, les nouvelles pousses sont suffisamment développées pour constituer les échantillons : les P.C.S., du printemps à l'aoûtement, sont donc mesurés sur des «pousses de l'année ». Puis, de septembre à décembre, les pousses 1979, aoûtées, sont considérées comme étant âgées d'une saison de végétation : elles se comportent déjà comme celles de début d'année.

Au cours de l'année, l'évolution du P.C.S. est régulière. Les toutes jeunes feuilles ou aiguilles, aux tissus alors très riches en cellulose et pauvres en lignine, présentent des P.C.S. de valeurs inférieures à ceux des feuilles âgées dont les tissus se sont enrichis en lignine par épaississement des parois cellulaires. L'augmentation des valeurs du P.C.S. et sa vitesse de variation caractérisent les espèces.

\subsection{Feuilles de sommités de bruyère arborescente}

Les P.C.S. évoluent entre $5588 \mathrm{cal} / \mathrm{g}$ en mai et $5981 \mathrm{cal} / \mathrm{g}$ en décembre $(23,4$ à $25 \mathrm{~kJ} / \mathrm{g}$ ), soit une amplitude de $393 \mathrm{cal} / \mathrm{g}$.

Deux ensembles se distinguent nettement: tissus ;

- les valeurs hivernales élevées liées à la période d'arrêt de l'élongation des

- les valeurs printanières et estivales, plus faibles, qui accompagnent la formation puis l'allongement des pousses de l'année 1979.

Un pic d'août, inexpliqué, précède de peu le passage d'un ensemble à l'autre. 


\subsection{Aiguilles terminales du pin d'Alep}

Les P.C.S. évoluent de $5115 \mathrm{cal} / \mathrm{g}$ en juillet à $5530 \mathrm{cal} / \mathrm{g}$ en février (de 21,4 à $23,1 \mathrm{~kJ} / \mathrm{g}$ ), soit une amplitude de $415 \mathrm{cal} / \mathrm{g}$.

Deux populations se distinguent:

- celle qui correspond aux P.C.S. des aiguilles «adultes» qui évoluent entre 5295 et $5530 \mathrm{cal} / \mathrm{g}$;

-- et celle, de juin à octobre, qui regroupe les P.C.S. des aiguilles de l'année 1979 , passant de 5115 à $5510 \mathrm{cal} / \mathrm{g}$.

En novembre et décembre, le site habituel étant inaccessible, les aiguilles ont été prólevées sur d'autres individus, ce qui explique vraisemblablement une certaine discontinuité dans les résultats, le P.C.S. d'une espèce variant parfois d'un sujet à l'autre.

\subsection{Sommités d'ajonc épineux}

Les P.C.S. varient très peu autour de $4994 \mathrm{cal} / \mathrm{g}(20,9 \mathrm{~kJ} / \mathrm{g})$. Le minimum est atteint en mai - $4926 \mathrm{cal} / \mathrm{g}$ - durant la période d'active élongation des tissus. Le maximum a lieu en hiver - décembre et janvier : $5093 \mathrm{cal} / \mathrm{g}$ - lors de l'arrêt de l'élongation et pendant la floraison.

La stabilité des résultats peut provenir de l'échantillonnage : l'absence de dimorphisme foliaire décelable à l'œil et la constance dans les formes architecturales conduisent à des lots apparemment homogènes d'un bout de l'année à l'autre.

\subsection{Feuilles de sommités d'arbousier}

Leurs P.C.S. évoluent entre $4848 \mathrm{cal} / \mathrm{g}$ et $5075 \mathrm{cal} / \mathrm{g}(20,3$ et $21,2 \mathrm{~kJ} / \mathrm{g})$ soit une amplitude de $227 \mathrm{cal} / \mathrm{g}$.

L'écart entre les P.C.S. de la période d'arrêt d'élongation et ceux de la période d'allongement est important malgré les valeurs de février et mars qui se détachent des valeurs hivernales pour des raisons inexpliquées. A noter que les P.C.S. d'avril et de mai ont été mesurés sur des feuilles de pousses de l'annće 1978.

Les valeurs atteintes en septembre - 5354 et $5137 \mathrm{cal} / \mathrm{g}$ - sont probablement erronées car leur différence de $217 \mathrm{cal} / \mathrm{g}$, quatre fois supérieure à la précision, aurait dû entraîner la réalisation d'une troisième mesure qui n'a pu être effectuée.

\subsection{Feuilles de sommités de chêne vert}

Leurs P.C.S. se situent aux alentours de $4840 \mathrm{cal} / \mathrm{g}(20,3 \mathrm{~kJ} / \mathrm{g})$ et deux pics se dessinent :

- en juin, période d'allongement des tissus, P.C.S. minimum de $4686 \mathrm{cal} / \mathrm{g}$;

— en octobre, période d'arrêt de l'élongation, P.C.S. maximum de $4979 \mathrm{cal} / \mathrm{g}$. 
En mai, le chêne vert porte à la fois des sommités de l'année 1978 et des sommités développées en 1979. La comparaison des P.C.S. des tissus anciens lignifiés 4830 et $4793 \mathrm{cal} / \mathrm{g}$ - avec ceux des jeunes tissus très cellulosiques - 4648 et $4660 \mathrm{cal} / \mathrm{g}$ - est très instructive.

L'espèce n'a pu être étudiée en septembre.

\subsection{Feuilles de sommités de chêne kermès}

Leurs P.C.S. évoluent autour de $4770 \mathrm{cal} / \mathrm{g}(20 \mathrm{~kJ} / \mathrm{g})$. Deux pics existent ici aussi, en mars et en août, difficiles à mettre en relation avec le stade phénologique.

Comme le chêne vert, le chêne kermès a la particularité de présenter en mai des sommités de l'année 1978 et de l'année 1979. Les P.C.S. des jeunes feuilles sont de 4485 et $4486 \mathrm{cal} / \mathrm{g}$ tandis que ceux des feuilles de l'année précédente sont de 4720 et $4728 \mathrm{cal} / \mathrm{g}$; l'écart est donc très important.

\section{2. - Spécificité du pouvoir calorifique supérieur}

Les variations des P.C.S. sont influencées par les stades phénologiques. Toutefois, l'amplitude de ces variations reste comprise entre 5 et 10 p. 100 des différentes moyennes arithmétiques. Au sein du tableau 3 ont été regroupés :

- la moyenne arithmétique des 22 ou 24 mesures effectuées

$$
\overline{\mathrm{x}}=\frac{1}{\mathrm{n}} \sum_{\mathrm{i}}^{\mathrm{i}}=1 \mathrm{n} \mathrm{x}_{\mathrm{i}}
$$

- l'estimation de l'écart-type : $\sigma$

$$
\sigma^{2}=\frac{1}{n-1} \sum_{i=1}^{i=n}\left(x_{i}-\bar{x}\right)^{2}
$$

- le coefficient de variation

$$
\mathrm{cv}=\sigma / \overline{\mathrm{x}}
$$

- les P.C.S. minimum, médian et maximum.

Un test de Newman et Keuls effectué à partir des données mensuelles permet d'établir au seuil de probabilité de 95 p. 100 le classement fourni au sein du tableau 3 : une même lettre regroupe les espèces dont les P.C.S. moyens ne sont pas statistiquement différents.

Le P.C.S. très élevé de la bruyère arborescente est remarquable : il est supérieur aux valeurs trouvées tant par W.A. Hougfi que par G. Heim. Il est même supérieur aux P.C.S. d'espèces signalées par W. LARCHER comme très énergétiques. 


\section{TABLEAU 3}

Spécificité du pouvoir calorifique supćrieur

High calorific value of different species

\begin{tabular}{|c|c|c|c|c|c|c|}
\hline Espèces étudiées & $\begin{array}{c}\text { Erica } \\
\text { arborea } \\
\\
\text { Bruyère } \\
\text { arbores- } \\
\text { cente }\end{array}$ & $\begin{array}{c}\text { Pinus } \\
\text { hale- } \\
\text { pensis } \\
\text { Pin } \\
\text { d'Alep }\end{array}$ & $\begin{array}{c}\text { Ulex } \\
\text { parvi- } \\
\text { florus } \\
\\
\text { Ajonc } \\
\text { épineux }\end{array}$ & $\begin{array}{l}\text { Arbutus } \\
\text { unedo } \\
\text { Arbou- } \\
\text { sier }\end{array}$ & $\begin{array}{l}\text { Quercus } \\
\text { ilex } \\
\text { Chêne } \\
\text { vert }\end{array}$ & $\begin{array}{c}\text { Quercus } \\
\text { coccifera } \\
\text { Chêne } \\
\text { kermès }\end{array}$ \\
\hline $\begin{array}{l}\text { Nombre de mesures } \ldots \ldots \\
\text { Moyenne des P.C.S. } \\
\text { cal } / \mathrm{g} \ldots \ldots \ldots \ldots \ldots \ldots \\
\mathrm{J} / \mathrm{g} \ldots \ldots \ldots \ldots \ldots \ldots\end{array}$ & $\begin{array}{r}5748 \\
24058\end{array}$ & $\begin{array}{r}5307 \\
22212\end{array}$ & $\begin{array}{r}4994 \\
20902\end{array}$ & $\begin{array}{r}4986 \\
20869\end{array}$ & $\begin{array}{r}4845 \\
20279\end{array}$ & $\begin{array}{r}4776 \\
19990\end{array}$ \\
\hline $\begin{array}{l}\text { Estimation de l'écart-type } \\
\qquad \begin{array}{c}\text { cal } / \mathrm{g} \\
\mathrm{J} / \mathrm{g} \ldots \ldots \ldots \ldots \ldots \ldots \ldots\end{array}\end{array}$ & $\begin{array}{l}115 \\
481\end{array}$ & $\begin{array}{l}149 \\
624\end{array}$ & $\begin{array}{r}45 \\
188\end{array}$ & $\begin{array}{r}67 \\
280\end{array}$ & $\begin{array}{r}72 \\
301\end{array}$ & $\begin{array}{l}110 \\
460\end{array}$ \\
\hline $\begin{array}{l}\text { Coefficient de variation } \ldots \\
\text { Médiane } \\
\quad \text { cal } / \mathrm{g} \quad \ldots \ldots \ldots \ldots \ldots \ldots \\
\mathbf{J} / \mathrm{g} \ldots \ldots \ldots \ldots \ldots \ldots\end{array}$ & $\begin{array}{r}5733 \\
23995\end{array}$ & $\begin{array}{r}5302 \\
22192\end{array}$ & $\begin{array}{r}4988 \\
20877\end{array}$ & $\begin{array}{r}5016 \\
20994\end{array}$ & $\begin{array}{r}4849 \\
20595\end{array}$ & $\begin{array}{r}4756 \\
19906\end{array}$ \\
\hline $\begin{array}{l}\text { Maximum } \\
\qquad \mathrm{cal} / \mathrm{g} \ldots \ldots \ldots \ldots \ldots \ldots \ldots \\
\mathbf{J} / \mathrm{g} \ldots \ldots \ldots \ldots \ldots\end{array}$ & $\begin{array}{r}5981 \\
25033\end{array}$ & $\begin{array}{r}5530 \\
23146\end{array}$ & $\begin{array}{r}5093 \\
21317\end{array}$ & $\begin{array}{r}5075 \\
21141\end{array}$ & $\begin{array}{r}4979 \\
20840\end{array}$ & $\begin{array}{r}5020 \\
21011\end{array}$ \\
\hline $\begin{array}{l}\text { Minimum } \\
\text { cal } / g \quad \ldots \\
\mathrm{J} / \mathrm{g}\end{array}$ & $\begin{array}{r}5588 \\
23386\end{array}$ & $\begin{array}{r}5115 \\
21409\end{array}$ & $\begin{array}{r}4926 \\
20618\end{array}$ & $\begin{array}{r}4848 \\
20291\end{array}$ & $\begin{array}{r}4686 \\
19613 \\
\end{array}$ & $\begin{array}{r}4540 \\
19002\end{array}$ \\
\hline $\begin{array}{l}\text { Amplitude } \\
\text { cal/g } \ldots \\
\mathrm{J} / \mathrm{g} \ldots\end{array}$ & $\begin{array}{r}393 \\
1645\end{array}$ & $\begin{array}{r}415 \\
1737\end{array}$ & $\begin{array}{l}167 \\
699\end{array}$ & $\begin{array}{l}227 \\
950\end{array}$ & $\begin{array}{r}293 \\
1226\end{array}$ & $\begin{array}{r}480 \\
2009\end{array}$ \\
\hline Significativité classement & $a$ & $\mathrm{~b}$ & $\mathrm{c}$ & $\mathrm{c}$ & $\mathrm{d}$ & $\mathrm{e}$ \\
\hline
\end{tabular}

Celui du pin d'Alep est du même ordre que celui mesuré par G. Heim - au printemps, 5393 contre $5377 \mathrm{cal} / \mathrm{g}$ - , mais il est supérieur aux valeurs fournies par W.A. Hough, pour des pins américains il est vrai.

Le P.C.S. de l'ajonc épineux ou de larbousier - environ $5000 \mathrm{cal} / \mathrm{g}$ $(20,9 \mathrm{~kJ} / \mathrm{g})$ - est comparable à celui de la lavande ou du pistachier lentisque donné par G. HEIM. 


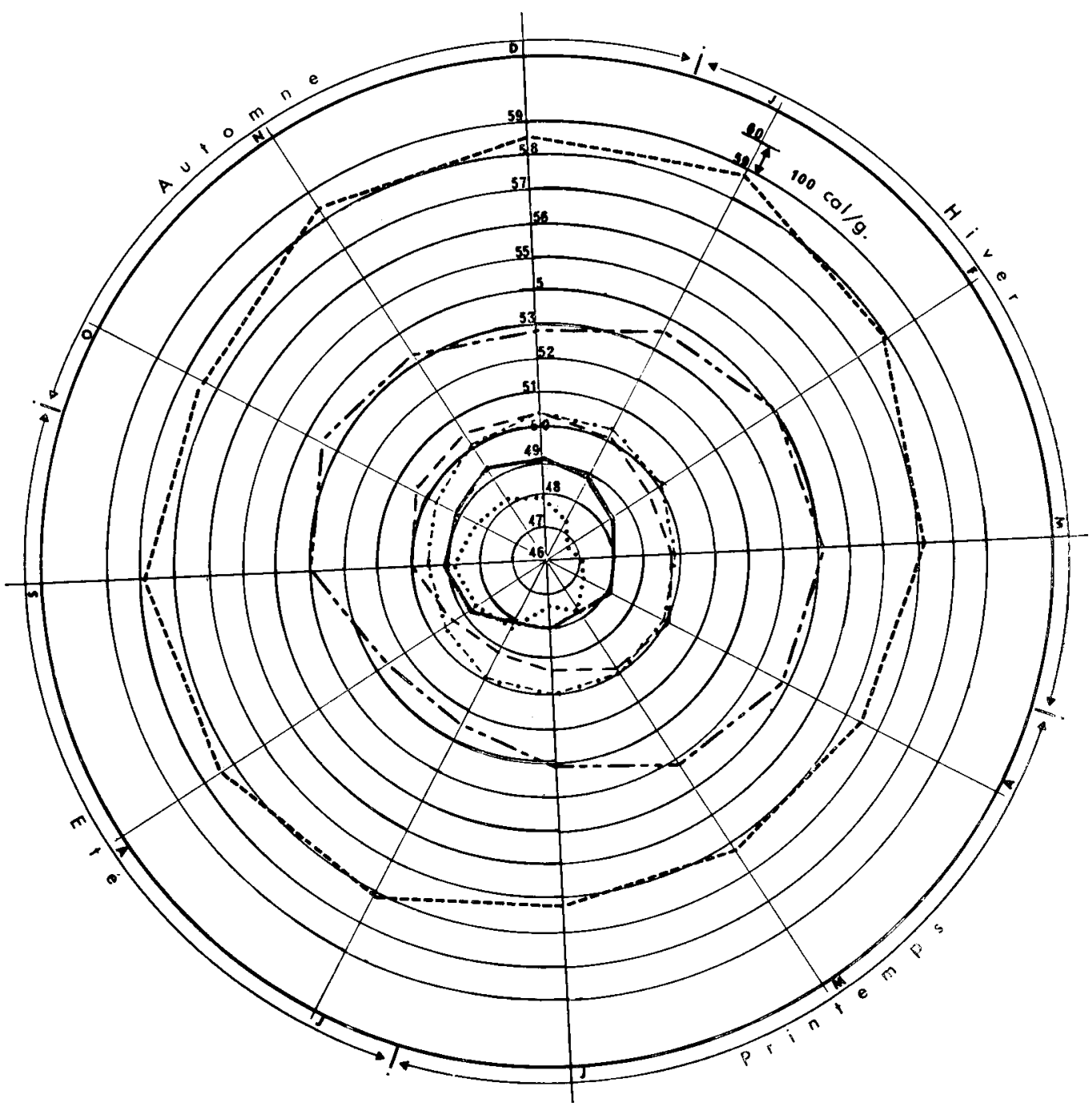

Fig. 2

Pouvoir calorifique supérieur

Calorific values «moving average»

Moyenne lissée, pas de : 3

Bruyère arborescente

Pin d'Alep

Ajonc épineux

Arbousier

Chêne vert

Chêne kermès 
Quant aux chênes, leurs P.C.S. sont très voisins aussi bien des valeurs trouvées par G. Heim que de celles fournies par W.A. Hough pour les chênes du Sud et de l'Est des U.S.A.

Tous ces P.C.S. sont indiqués dans le tableau en annexe.

La figure $\mathrm{n}^{\circ} 2$ obtenue par lissage des données de la figure $\mathrm{n}^{\circ} 1$ met bien en évidence la spécificité des variations du P.C.S. Le vecteur porté sur l'axe «février», par exemple, est d'un module égal à la moyenne arithmétique des P.C.S. des mois de janvier, février et mars.

Les pouvoirs calorifiques supérieurs

- de la bruyère arborescente :

- diminuent de la fin de la floraison (février-mars) à l'arrêt de l'élongation (juin-juillet),

- se stabilisent durant le repos estival,

- augmentent durant la floraison (novembre à janvier) ;

- du pin d'Alep :

- diminuent de mai à septembre (feuillaison et repos estival),

- se stabilisent en automne,

- augmentent en hiver jusqu'au débourrement ;

- de lajonc épineux :

- sont très stables toute l'année,

- diminuent légèrement lors de la reprise automnale de la croissance,

- augmentent légèrement lors de la floraison (janvier-février) ;

- de l'arbousier :

- diminuent durant la phase active de la croissance printanière,

- atteignent leurs maxima lors de la floraison et de la fructification (novembrejanvier) ;

- du chêne vert :

- diminuent durant la croissance printanière,

- atteignent leurs maxima durant le repos hivernal après le second aoûtement;

- du chêne kermès :

- sont à leur maxima avant la reprise automnale de la végétation,

- conservent des valeurs relativement basses durant l'élongation.

\section{3. - Influence de la composition des tissus sur le P.C.S.}

\subsection{La matière organique}

A plusieurs reprises, les écarts importants entre les P.C.S. des pousses en formation riches en cellulose et ceux des pousses âgées d'une saison de végétation riches en lignine ont été signalés. Les mesures étant effectuées sur de la matière sèche, ainsi 
qu'indiqué au $\S 2.2$., les teneurs en eau, au demeurant fort différentes, n'expliquent en rien les écarts.

Par contre, et de nombreux auteurs l'ont déjà signalé, les potentiels énergétiques de la lignine - $6100 \mathrm{cal} / \mathrm{g}(25,5 \mathrm{~kJ} / \mathrm{g})$ - et des celluloses — de l'ordre de $4000 \mathrm{cal} / \mathrm{g}$ $(16,7 \mathrm{~kJ} / \mathrm{g})$ - suffisent à l'explication.

\subsection{Les cendres totales}

La teneur a été mesurée par passage à l'étuve à $105^{\circ} \mathrm{C}$ suivi d'une minéralisation au four à mouffle à $450^{\circ} \mathrm{C}$; elle s'exprime en pourcentage de la matière sèche.

Des teneurs spécifiques peuvent être déterminées; elles permettent de calculer, par application de la formule donnée au $\S 2.1$ ce que W.A. Hough (1969) dénomme "ash-free caloric value » (le P.C.S. de la partie sans cendres, de la partie non minérale).

\section{Tableau 4}

Teneurs en cendres, P.C.S. et P.C.S. de la matière organique spécifiques Ash content, high calorific (*) value and ash-free calorific value

\begin{tabular}{|c|c|c|c|c|c|}
\hline \multirow{2}{*}{ Espèces } & \multirow{2}{*}{$\begin{array}{l}\text { Teneur } \\
\text { en cendres } \\
(\%)\end{array}$} & \multicolumn{2}{|c|}{ P.C.S. } & \multicolumn{2}{|c|}{ P.C.S. org. } \\
\hline & & $\mathrm{Cal} / \mathrm{g}$ & $\mathrm{J} / \mathrm{g}$ & $\mathrm{Cal} / \mathrm{g}$ & $\mathrm{J} / \mathrm{g}$ \\
\hline Bruyère arborescente & 2,42 & 5748 & 24058 & 5841 & 24657 \\
\hline Pin d'Alep $\ldots \ldots \ldots$ & 3,20 & 5307 & 22212 & 5482 & 22945 \\
\hline Ajonc épineux & 2,34 & 4994 & 20902 & 5114 & 21405 \\
\hline Arbousier $\ldots$ & 4,15 & 4986 & 20969 & 5202 & 21773 \\
\hline Chêne vert .. & 4,05 & 4845 & 20279 & 5050 & 21137 \\
\hline Chêne kermès & 4,20 & 4776 & 19990 & 4991 & 21890 \\
\hline
\end{tabular}

(*) Longtemps les spécialistes anglo-saxons ont parlé de caloric value; il semblerait qu'ils utilisent maintenant le terme de calorific value (CF norme expérimentale AFNOR x 65-020 de décembre 1978).

\section{4. - Liaison entre le P.C.S. et l'inflammabilité}

La présente étude confirme les résultats des travaux des chercheurs du C.S.T.B. (*). Le P.C.S. est une propriété fondamentale du combustible en relation directe avec sa composition tandis que l'inflammabilité est essentiellement liée aux caractéristiques de surface du combustible.

Comme pour les bois tropicaux (J. DoaT \& J.Ch. VAletTe, 1980) ces deux paramètres sont indépendants.

(*) Centre Scientifique et Technique du Bâtiment, Champs-sur-Marne. 
Deux exemples : la bruyère arborescente et le pin d'Alep sont deux espèces très inflammables et aux P.C.S. élevés quoique statistiquement différents. Le chêne vert est autant si ce n'est plus inflammable que le pin d'Alep, son P.C.S. est très nettement plus faible.

\section{4. - Conclusion}

Cette étude a permis de déterminer les valeurs spécifiques du P.C.S. sur une année. Elle autorise donc le calcul, dans les conditions expérimentales du *banc d'essais thermique» de Valabre ou dans les conditions réelles d'un incendie, de la puissance du front de feu, quantité d'énergie dégagée par unité de temps et de longueur du front de feu.

Toutefois, l'incendie de forêt ne consomme pas seulement les sommités des végétaux : ramilles, branches et parfois les troncs participent au phénomène. Il serait intéressant de poursuivre ces études afin de mettre en évidence les différences susceptibles d'exister entre les P.C.S. des sommités, des ramilles, des branches et des troncs. Ces connaissances conduiraient à la détermination, pour un végétal ligneux donné, d'un P.C.S. moyen pondéré.

Les valeurs relativement élevées du P.C.S. rendent envisageable l'utilisation d'au moins quelques espèces comme source d'énergie. Les difficultés de la récolte ne sont pas pour autant résolues : 25 à 30 tonnes de matières sèches au mieux récupérables à l'hectare, dans des terrains généralement difficiles d'accès. Aux problèmes d'exploitation et de débusquage, sajoutent les conditions de séchage avant transport ou son conditionnement sur place de façon à économiser de l'énergie.

Il y a souvent loin de la théorie à la pratique : la récupération de l'énergie, potentiellement présente, d'une phytomasse constituée de broussailles et d'arbustes en est un bel exemple.

Reçu pour publication en décembre 1980.

\section{Summary}

High calorific value of some mediterranean forest species

High calorific value (P.C.S.) represents the maximum of energy released by combustion. It allows the computation of the forest fire heat release.

On a dry matter basis or on an ash-free basis datas group the species in classes :

- leaves of Erica arborea L.,

- needles of Pinus halepensis Mill.,

- leaves of Arbutus unedo L. and top-end sprouts of Ulex parviflorus Pourret,

- leaves of Quercus ilex L. and Quercus coccifera L.

High calorific values rise with the lignification.

High calorific values of these mediterranean species are higher than these of more temperate species, or at least equal to these. 


\section{Références bibliographiques}

Bong D.A., Kiceniuk J.W., 1968. Protein and caloric content of lodge pole pine needles. For. Chron., 44 (4), 28-31.

BLIss L.C., 1962. Caloric and lipid content in alpine tundra plants. Ecology, 43 (4), 753-757.

Dagnelie P., 1978. Théorie et méthodes statistiques. 2 tomes. 3" édition. Presses agronomiques de Gembloux, 378 et $463 \mathrm{p}$.

Doat J., 1977. Le pouvoir calorifique supérieur des bois tropicaux. Bois For. Trop. (Nogentsur-Marne), 172, 33-55.

Doat J., Valette J.Ch., 1980. L'inflammabilité de quelques bois tropicalx. Bois. For. Trop. (Nogent-sur-Marne), 193.

Golley F.B., 1969. Caloric value of wet tropical forest vegetation. Ecology, 50 (3), 517-519.

Gorham E., SANGER J., 1967. Caloric value of organic matter in woodland, swamp and lake soils. Ecology, 48 (3), 492-494.

HEIM G., 1974. L'utilité du concept de valeur énergétique en écologie : une étude basée sur des mesures effectuées sur des plantes méditerranéennes. Oecol. Plant., 9 (3), 281-286.

Hough W.A., 1969. Caloric value of some forest fuels of Southern United States. U.S.D.A. Forest Service Research, Note SE-120, Southern Forest Experiment Station, Asheville, North Carolina, 5 p..

Larcher W., Schmidt L., Tscharger A., 1973. Starke Fettspeicherung und hoher Kaloriengehalt bei Loiseleuria procumbens (L.) Desv. Oecol. Plant., 8 (4), 377-383.

Ovington J.D., Heitkamp D., 1960. The accumulation of energy in forest plantations in Britain. Ecology, 41, 639-646.

Sмrтн E.E., 1972. Heat release rate of building materials, Ignition. Heat Release and Noncombustibility of Materials, A.S.T.M. S.T.P. 502, 119-134.

VALET TE J.Ch., 1980. Composition minérale d'espèces méditerranéennes (Document à paraître).

Valette J.Ch., Clément A., Delabraze P., 1979. Inflammabilité d'espèces méditerranéennes. Tests rapides, campagne été 1978. Document interne 79-3 de la Station de Sylviculture méditerranéenne, Avignon, 39 p. + annexes.

Verduin J., 1972. Caloric content and avaible energy in plant matter. Ecology, 53 (5). 


\section{ANNEXE}

Quelques valeurs de P.C.S. tirées de la littérature

\begin{tabular}{|c|c|c|c|}
\hline \multirow{2}{*}{ Matériels étudiés } & \multicolumn{2}{|c|}{ P.C.S. } & \multirow{2}{*}{ Auteurs } \\
\hline & $\mathrm{cal} / \mathrm{g}$ & $\mathrm{kJ} / \mathrm{g}$ & \\
\hline \multicolumn{4}{|l|}{ Combustibles fossiles } \\
\hline Tourbe $\ldots \ldots \ldots$ & 5000 & 20,9 & DOAT \\
\hline Lignite $\ldots \ldots \ldots \ldots \ldots \ldots \ldots$ & 6250 & 26,2 & DOAT \\
\hline Anthracite $\ldots \ldots \ldots \ldots \ldots \ldots \ldots \ldots$ & 7250 & 30,3 & DOAT \\
\hline Pétrole $\ldots \ldots \ldots \ldots \ldots \ldots \ldots \ldots \ldots \ldots \ldots \ldots \ldots \ldots$ & 11000 & 46,0 & DOAT \\
\hline \multicolumn{4}{|l|}{ Eléments chimiques } \\
\hline Carbone ......... & 8100 & 33,9 & DOAT \\
\hline Hydrogène . & 34000 & 142,3 & DOAT \\
\hline \multicolumn{4}{|l|}{ Matières organiques } \\
\hline Celluloses $\ldots . .$. & 3700 & 15,5 & VERDUIN \\
\hline Celluloses . & 4070 & 17,0 & DOAT \\
\hline Protéines ....... & 5700 & 23,9 & VERDUIN \\
\hline Carbohydrates $\ldots \ldots \ldots \ldots \ldots \ldots \ldots \ldots$ & 5100 & 21,3 & VERDUIN \\
\hline 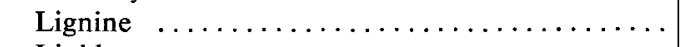 & 6100 & 25,5 & DOAT \\
\hline Lipides $\quad \ldots \ldots \ldots \ldots \ldots \ldots \ldots \ldots \ldots \ldots \ldots \ldots \ldots$ & 9300 & 38,9 & VERDUIN \\
\hline \multicolumn{4}{|l|}{ Végétaux } \\
\hline Chêne (bois) & 4670 & 19,5 & DOAT \\
\hline Hêtre (bois) ...... & 4680 & 19,6 & DOAT \\
\hline Epicéa (bois) $\ldots \ldots \ldots \ldots \ldots \ldots \ldots \ldots \ldots \ldots \ldots \ldots \ldots \ldots \ldots$ & 4770 & 20,0 & DOAT \\
\hline Pin maritime (bois) $\ldots \ldots \ldots \ldots \ldots \ldots \ldots$ & 4820 & 20,2 & DOAT \\
\hline \multicolumn{4}{|l|}{ Espèces arborées nord-américaines } \\
\hline Quercus virginiana Mill. (feuilles) $\ldots \ldots \ldots \ldots$ & 4708 & 19,7 & Hough \\
\hline Quercus obtusiloba Michx. (feuilles) .... & 4882 & 20,4 & Hough \\
\hline Quercus laevis (feuilles) $\ldots \ldots \ldots \ldots \ldots \ldots$ & 4893 & 20,5 & Hough \\
\hline Populus tremulö̈des Michx. (ramilles) ...... & 4864 & 20,4 & HovGH \\
\hline Populus tremuloïdes Michx. (feuilles) ....... & 5053 & 21,1 & HougH \\
\hline Larix decidua Miller (aiguilles) $\ldots \ldots \ldots \ldots$ & 4608 & 19,3 & Hough \\
\hline Larix laricina K. Koch (aiguilles) & 4637 & 19,4 & Hough \\
\hline Picea glauca Voss. (aiguilles) $\ldots \ldots \ldots \ldots \ldots$ & 4700 & 19,7 & HougH \\
\hline Picea glauca Voss. (ramilles) $\ldots \ldots \ldots \ldots \ldots$ & 5070 & 21,2 & Hough \\
\hline Picea mariana B.S.P. (aiguilles) $\ldots \ldots \ldots$ & 4926 & 20,6 & Hough \\
\hline Picea mariana B.S.P. (ramilles) $\ldots \ldots \ldots \ldots$ & 5125 & 21,5 & HOUGH \\
\hline Abies balsamea Mill. (aiguilles) $\ldots \ldots \ldots \ldots$ & 5060 & 21,2 & Hough \\
\hline Abies balsamea Mill. (ramilles) ..... & 5040 & 21,1 & HoUGH \\
\hline Pinus clausa Vasey jeun. (aiguilles) $\ldots \ldots \ldots$ & 4865 & 20,4 & Hough \\
\hline Pinus clausa Vasey viei. (aiguilles) $\ldots \ldots \ldots$ & 5443 & 22,8 & Hough \\
\hline Pinus clausa Vasey (ramilles) $\ldots \ldots \ldots \ldots$ & 5293 & 22,3 & Hough \\
\hline Pinus banksiana Lamb. (aiguilles) $\ldots \ldots \ldots \ldots$ & 5102 & 21,4 & Hough \\
\hline Pinus banksiana Lamb. (ramilles) & 5176 & 21,7 & HOUGH \\
\hline Pinus elliotii Engel. (aiguilles) .. & 5206 & 21,8 & Hough \\
\hline Pinus concorta Dougl. (aiguilles) & 5120 & 21,4 & BOAG \& KicENIUK \\
\hline Pinus cembroïdes edulis (aiguilles) ......... & 5000 & 20,9 & BOAG \& KicENIUK \\
\hline
\end{tabular}




\begin{tabular}{|c|c|c|c|}
\hline \multirow{2}{*}{ Matériels étudiés } & \multicolumn{2}{|c|}{ P.C.S. } & \multirow{2}{*}{ Auteurs } \\
\hline & $\mathrm{cal} / \mathrm{g}$ & $\mathrm{kJ} / \mathrm{g}$ & \\
\hline Espèces alpines et de la toundra & & & \\
\hline $\begin{array}{l}\text { Loiseleuria procumbens }(\mathbf{L} .) \text { Desv. } \\
\text { Jeunes feuilles } \ldots \ldots \ldots \ldots \ldots \ldots \ldots \ldots \ldots \ldots \ldots \ldots \ldots \\
\text { Vieilles feuilles } \ldots \ldots \ldots \ldots \ldots \ldots \ldots \ldots\end{array}$ & $\begin{array}{l}5570 \\
5650\end{array}$ & $\begin{array}{l}23,3 \\
23,6\end{array}$ & $\begin{array}{l}\text { LARCHER et al. } \\
\text { LARCHER et al. }\end{array}$ \\
\hline Empetrum hermaphroditum (feuilles) ......... & 5560 & 23,3 & Bliss \& HadLEY \\
\hline Diapensia lapponica (feuilles) ............. & 5640 & 23,6 & Bliss \& HadLey \\
\hline Ledum groenlandicum (feuilles) $\ldots \ldots \ldots \ldots$ & 5650 & 23,6 & BLISS \& HADLEY \\
\hline Espèces méditerranéennes & & & \\
\hline $\begin{array}{l}\text { Acer monspessulanum } \mathbf{L} . \\
\text { Pousses nouvelles } \ldots \ldots \ldots \ldots \ldots \ldots \ldots \ldots \ldots \ldots \ldots \ldots \\
\text { Tiges lignifiées } \ldots \ldots \ldots \ldots \ldots \ldots \ldots\end{array}$ & $\begin{array}{l}4708 \\
4541\end{array}$ & $\begin{array}{l}19,7 \\
19,0\end{array}$ & $\begin{array}{l}\text { HeIM } \\
\text { HeIM }\end{array}$ \\
\hline $\begin{array}{l}\text { Cistus monspelliensis } \mathrm{L} . \\
\text { Pousses nouvelles } \ldots \ldots \ldots \ldots \ldots \ldots \ldots \ldots \ldots \ldots \ldots \ldots \ldots \\
\text { Feuilles } \ldots \ldots \ldots \ldots \ldots \ldots \ldots \ldots \ldots\end{array}$ & $\begin{array}{l}5425 \\
4756\end{array}$ & $\begin{array}{l}22,7 \\
19,9\end{array}$ & $\begin{array}{l}\text { HeIM } \\
\text { HeIM }\end{array}$ \\
\hline Juniperus oxycedrus L. (pousses nouvelles) .. & 5138 & 21,5 & HEIM \\
\hline Lavandula stoechas L. (pousses nouvelles) ... & 5019 & 21,0 & HEIM \\
\hline Morus alba L. (feuilles) $\ldots \ldots \ldots \ldots \ldots$ & 4493 & 18,8 & HEIM \\
\hline Phyllyrea angustifolia L. (pousses nouvelles) .. & 5186 & 21,7 & HEIM \\
\hline 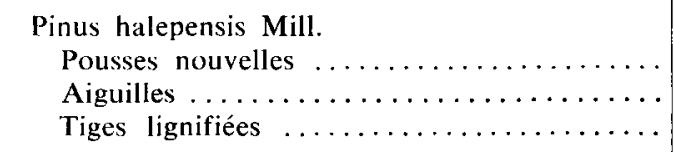 & $\begin{array}{l}5234 \\
5377 \\
5138\end{array}$ & $\begin{array}{l}21,9 \\
22,5 \\
21,5\end{array}$ & $\begin{array}{l}\text { HEIM } \\
\text { HEIM } \\
\text { HEIM }\end{array}$ \\
\hline Pistacia lentiscus L. (feuilles) $\ldots \ldots \ldots \ldots \ldots$ & 4911 & 20,6 & HEIM \\
\hline Pistacia terebinthus $L$. (pousses nouvelles) ... & 4684 & 19,6 & HeIM \\
\hline $\begin{array}{l}\text { Quercus coccifera } \mathrm{L} . \\
\text { Pousses nouvelles } \ldots \ldots \ldots \ldots \ldots \ldots \ldots \ldots \ldots \ldots \ldots \\
\text { Feuilles } \quad \ldots \ldots \ldots \ldots \ldots \ldots \ldots \ldots\end{array}$ & $\begin{array}{l}4469 \\
4744\end{array}$ & $\begin{array}{l}18,7 \\
19,9\end{array}$ & $\begin{array}{l}\text { HEIM } \\
\text { HEIM }\end{array}$ \\
\hline $\begin{array}{l}\text { Quercus ilex } \mathrm{L} . \\
\text { Pousses nouvelles } \ldots \ldots \ldots \ldots \ldots \ldots \ldots \ldots \ldots \ldots \\
\text { Feuilles } \quad \ldots \ldots \ldots \ldots \ldots \ldots \ldots \ldots \ldots\end{array}$ & $\begin{array}{l}4851 \\
4815\end{array}$ & $\begin{array}{l}20,3 \\
20,2\end{array}$ & $\begin{array}{l}\text { HEIM } \\
\text { HEIM }\end{array}$ \\
\hline $\begin{array}{l}\text { Rosmarinus officinalis } \mathbf{L} . \\
\text { Pousses nouvelles } \ldots \ldots \ldots \ldots \ldots \ldots \ldots \ldots \ldots \ldots \\
\text { Feuilles } \ldots \ldots \ldots \ldots \ldots \ldots \ldots \ldots \ldots \ldots\end{array}$ & $\begin{array}{l}5473 \\
5449\end{array}$ & $\begin{array}{l}22,9 \\
22,8\end{array}$ & $\begin{array}{l}\text { HEIM } \\
\text { HEIM }\end{array}$ \\
\hline
\end{tabular}

